\title{
Características morfoanatômicas de folhas de cana-de-açúcar sob efeito residual de maturadores ${ }^{1}$
}

\section{Morphoanatomic characteristics of the sugarcane's leaves under residual effect after ripeners application}

\section{Ronaldo da Silva Viana ${ }^{2}$; Paulo Alexandre Monteiro Figueiredo³; Lucas Aparecido Manzani Lisboa $^{4}$; Isabô Melina Pascoaloto ${ }^{5}$}

Resumo - O objetivo do estudo foi avaliar os efeitos residuais de maturadores nas características morfoanatômicas de folhas da cana-de-açúcar. $\mathrm{O}$ delineamento experimental utilizado foi em blocos casualizados no esquema de parcelas subdivididas. As parcelas foram constituídas pelas variedades de cana-de-açúcar (SP80-3280 e SP80-1842) e as subparcelas dos diferentes maturadores: Curavial 20 g p.c. ha ${ }^{-1}$ (sulfometuron-methyl); Curavial $20 \mathrm{~g}$ p.c. $\mathrm{ha}^{-1}+$ Roundup 0,15 L ha ${ }^{-1}$ (sulfometuron-methyl + glyphosate); Ethrel 0,67 L ha-1 (etephon); Ethrel 0,67 $\mathrm{L} \mathrm{ha}^{-1}$ + Roundup 0,15 $\mathrm{L} \mathrm{ha}^{-1}$ (etephon + glyphosate); Moddus 0,80 $\mathrm{L} \mathrm{ha}^{-1}$ (ethyltrinexapac); MTD 1,0 L ha-1 (Compostos de radicais carboxílicos orgânicos); MTD 1,0 L ha ${ }^{-1}+$ Roundup 0,15 L ha-1 (Compostos de radicais carboxílicos orgânicos + glyphosate); Roundup 0,15 $\mathrm{L} \mathrm{ha}^{-1}$ (glyphosate); e Testemunha. Foram mensurados os seguintes parâmetros quantitativos dos tecidos: espessura da epiderme da face adaxial; espessura da epiderme da face abaxial; espessura do mesofilo; espessura do limbo; diâmetro do xilema; e diâmetro do floema. De maneira geral, as características morfoanatômicas foliares de cana-de-açúcar apresentaram maiores valores médios para o efeito residual do maturador Ethrel (etephon). A variedade SP80-1842 exibiu os maiores valores para a maioria das características estudadas.

Palavras-chaves: fitorreguladores vegetal; morfologia; Saccharum spp.

Abstract - The aim of this study was to evaluate the residual effects regarding the maturing on morphoanatomic characteristics relating the sugarcane's leaves. The experimental design was a randomized block in a split plot. The plots were constituted by the following varieties of sugarcane (SP80-3280 and SP80-1842) and the subplots of different maturings: Curavial $20 \mathrm{~g}$ bw $\mathrm{ha}^{-1}$ (sulfomethuron-methyl); Curavial 20g bw ha-1 + Roundup $0.15 \mathrm{~L} \mathrm{ha}^{-1}$ (sulfomethuron-methyl + glyphosate); Ethrel 0.67 $\mathrm{L} \mathrm{ha}^{-1}$ (etephon); Ethrel 0.67 $\mathrm{L} \mathrm{ha}^{-1}+$ Roundup 0.15 L ha-1 (etephon glyphosate +); Moddus $0.80 \mathrm{~L} \mathrm{ha}^{-1}$ (ethyl-trinexapac); MTD 1,0 $\mathrm{L} \mathrm{ha}^{-1}$ (Compounds of organic

1 Recebido para publicação em 11/12/2015 e aceito em 13/02/2016.

2 Professor Assistente Doutor da FCAT - Faculdade de Ciências Agrárias e Tecnológicas da Unesp de Dracena - Email: ronaldo@dracena.unesp.br.

3Professor Adjunto da FCAT - Faculdade de Ciências Agrárias e Tecnológicas da Unesp de Dracena - E-mail: paulofigueiredo@dracena.unesp.br.

4 Doutorando do Programa de Pós-Graduação em Ciência e Tecnologia Animal da FCAT - Faculdade de Ciências Agrárias e Tecnológicas da Unesp de Dracena e FEIS - Faculdade de Engenharia da Unesp de Ilha Solteira - E-mail: lisboa@dracena.unesp.br.

5 Mestranda do Programa de Pós-graduação da Faculdade de Engenharia da Unesp de Ilha Solteira - E-mail: isabomelina@gmail.com. 
carboxylic radicals); MTD 1.0 $\mathrm{L} \mathrm{ha}^{-1}+$ Roundup $0.15 \mathrm{~L} \mathrm{ha}^{-1}$ (Compounds of organic carboxylic radicals + glyphosate); Roundup $0.15 \mathrm{~L} \mathrm{ha}^{-1}$ (glyphosate); and check without application. The following quantitative parameters of the tissues were measured: thickness of the epidermis of the adaxial face; thickness of the epidermis of the abaxial surface; thickness of the mesophyll; blade thickness; diameter of the xylem; diameter and phloem. In general, the foliar morphoanatomic characteristics of the sugarcane showed higher average values for the residual effect of Ethrel maturing (etephon). The SP80-1842 variety demonstrated the highest values for most characteristics under study.

Keywords: plant phytoregulators; morphology; Saccharum spp.

\section{Introdução}

Com o aumento na demanda de combustíveis renováveis, a indústria sucroalcooleira tem tido, como principal desafio, o aumento da produtividade agroindustrial da cana-de-açúcar, além da redução dos custos de produção. A utilização de maturadores químicos na cultura da canade-açúcar é uma realidade cada vez mais frequente, uma vez que facilitam o planejamento de colheita e aumentam a produtividade agroindustrial da cana-de-açúcar. No Brasil, têm sido utilizados, preferencialmente, os maturadores classificados como retardadores de crescimento, como o etephon, ethyl-trinexapac e os inibidores do crescimento, como o glyphosate e o sulfometuron-methyl (Leite, 2011).

Sob o ponto de vista econômico, a cana-de-açúcar é considerada madura, ou em condições de ser industrializada, a partir do momento em que apresentar um teor mínimo de sacarose, com Pol acima de $13 \%$ do peso do colmo (Benedini e Júnior, 2009). Entretanto, a cultura exige baixa temperatura durante a maturação para que ocorra redução no ritmo do desenvolvimento vegetativo e maior acúmulo de sacarose nos colmos (Scarpari e Beauclair, 2004). Como tais condições nem sempre são obtidas de forma natural em campo no período necessário para atender à logística industrial, o uso de maturadores tem se tornado imprescindível para o setor sucroenergético.

A aplicação de maturadores no início da safra pode resultar em incremento do teor de sacarose e antecipação da maturação; quando aplicados no meio da safra pode promover a elevação do teor de sacarose em regiões nas quais o inverno é chuvoso e quando aplicados no final da safra promovem o impedimento da retomada de crescimento da planta, mantendo o teor de sacarose ou diminuindo sua velocidade de redução (Silva, 2012).

Os reguladores vegetais, classificados como maturadores, são compostos químicos capazes de modificar a morfologia e fisiologia vegetal podendo ocasionar modificações qualitativas e quantitativas na produção (Leite, 2009). Tais compostos favorecem o acúmulo mais uniforme de açúcares nos entrenós da região apical, que normalmente são imaturos. São aplicados com a finalidade de antecipar o processo de maturação, promover melhorias na qualidade da matéria-prima a ser processada, otimizar os resultados agroindustriais e econômicos, além de auxiliar no planejamento da safra (Caputo, 2008).

Todavia, alterações visíveis a olho nu são consequências de modificações das estruturas dos tecidos dérmicos, fundamentais ou vasculares das plantas. O conhecimento dessas alterações morfoanatômicas de cada composto na planta é importante para a escolha do melhor produto a ser utilizado, conforme Castro et al. (2009) e Silva et al. (2015).

Alterações na espessura do mesófilo, por exemplo, podem influenciar na quantidade ou qualidade das substâncias produzidas, o que interfere diretamente na produtividade das culturas, segundo Castro et al. (2009).

Na composição do mesofilo encontra-se o parênquima clorofiliano, que além de ser 
responsável pela realização da fotossíntese também possui substâncias nutritivas que permitem o metabolismo vital às plantas, de acordo com Figueiredo et al. (2013). Desse modo, o objetivo deste trabalho foi avaliar as características morfoanatômicas de folhas de cana-de-açúcar sob efeito residual da aplicação de maturadores.

\section{Material e Métodos}

O ensaio foi conduzido na fazenda Santo Antônio Ubasa, pertencente ao GRUPO COSAN - Unidade Barra (Usina da Barra), localizada no município de Igaraçu do Tietê, SP. O solo da área experimental era um Latossolo Vermelho Férrico eutrófico (Embrapa, 2013), com topografia semiplana.

O clima predominante na região é o Aw (Köppen), caracterizado como tropical úmido, com estação chuvosa no verão e seca no inverno, temperatura média anual de $21,6^{\circ} \mathrm{C}$, umidade relativa média de $70 \%$ (extremos de $77 \%$ em fevereiro e $59 \%$ em agosto) e média pluviométrica anual de $1.344 \mathrm{mmO}$ delineamento experimental utilizado foi o em blocos ao acaso, com quatro repetições. Os tratamentos foram arranjados em esquema de parcelas subdivididas, sendo as parcelas constituídas de duas variedades de cana-deaçúcar (SP80-3280 e SP80-1842) e as subparcelas constituídas com aplicação de diferentes maturadores.

Os tratamentos foram: C - Curavial $20 \mathrm{~g}$ p.c. ha ${ }^{-1}$ (sulfometuron-methyl); C+R Curavial 20g p.c. ha ${ }^{-1}+$ Roundup $0,15 \mathrm{~L} \mathrm{ha}^{-1}$ (sulfometuron-methyl + glyphosate); E - Ethrel $0,67 \mathrm{~L} \mathrm{ha}^{-1}$ (etephon); E+R - Ethrel 0,67 $\mathrm{L} \mathrm{ha}^{-1}$ + Roundup 0,15 L ha-1 (etephon + glyphosate); M - Moddus 0,80 $\mathrm{L} \mathrm{ha}^{-1}$ (ethyl-trinexapac); MTD - MTD 1,0 L ha-1 (Compostos de radicais carboxílicos orgânicos); MTD + R - MTD 1,0 L ha ${ }^{-1}+$ Roundup 0,15 $\mathrm{L} \mathrm{ha}^{-1}$ (Compostos de radicais carboxílicos orgânicos + glyphosate); $\mathrm{R}$ - Roundup 0,15 L ha-1 (glyphosate); e Test Testemunha.
Cada parcela experimental foi formada de 7 linhas espaçadas em 1,40 m entre si, e 10 $\mathrm{m}$ de comprimento. As duas linhas laterais foram consideradas bordadura. As 5 linhas centrais consideradas como área útil.

O plantio da cana-de-açúcar ocorreu em 14 de fevereiro de 2007, durante o qual foram aplicados $2000 \mathrm{~kg} \mathrm{ha}^{-1}$ de calcário e $600 \mathrm{~kg} \mathrm{ha}^{-1}$ da fórmula 10-25-25 conforme exigido pela cultura com base na análise de solo realizada na área. A adubação de soqueira do quarto corte consistiu da aplicação de $322 \mathrm{~kg} \mathrm{ha}^{-1}$ da fórmula 33-00-00 e 0,150 $\mathrm{m}^{3} \mathrm{ha}^{-1}$ de vinhaça. O experimento foi instalado em 16 de março de 2011 em cana soca de quarto corte, sendo os maturadores aplicados no início da safra agrícola.

A aplicação dos maturadores químicos foi realizada utilizando-se um pulverizador de $\mathrm{CO}_{2}$ pressurizado, com uma barra de $6 \mathrm{~m}$ de comprimento, com 6 pontas AXI 11002 espaçados em $0,5 \quad \mathrm{~m}$, aplicando simultaneamente em duas linhas. A barra foi colocada horizontalmente apoiada sobre outras duas barras verticais que mantinham a barra pulverizadora a $50 \mathrm{~cm}$ acima do nível da cultura. A pressão utilizada foi de 40 libras/pol ${ }^{2}$ com um volume de calda de $200 \mathrm{~L}$ $\mathrm{ha}^{-1}$. A aplicação iniciou-se às 08h:00min e terminou às $11 \mathrm{~h}: 00 \mathrm{~min}$ horas, período que houve pouca ocorrência de ventos, temperatura ao redor de 25 a $30^{\circ} \mathrm{C}$ e a umidade relativa entre $60-80 \%$.

A colheita da soca do quarto ano da cana-de-açúcar ocorreu em 16 de junho de 2011. A rebrota da cana soca ocorreu em novembro de 2011 e após 60 dias da rebrota foram coletados fragmentos de aproximadamente $5 \mathrm{~cm}$ de folha das plantas de cana-de-açúcar em cada parcela, retirados da porção mediana da primeira folha completamente expandida a partir do ápice da cana. Os fragmentos foliares foram fixados em solução F.A.A.50 (formaldeído 37\%, ácido acético glacial e etanol $50 \%$ na proporção de 1:1:18 - V/V) e após 24 horas, foram lavados em etanol $50 \%$ e depois armazenados em 
etanol $70 \%$ até a data de realização das análises, segundo Kraus e Arduim (1999). Todos os fragmentos de folhas receberam os procedimentos pertinentes à desidratação, diafanização, inclusão e emblocagem.

Com auxílio de um micrótomo de mesa Leica contendo lâminas de aço, foram realizadas secções transversais de $8 \mu \mathrm{m}$ nos fragmentos foliares que continham a nervura central. Os fragmentos sofreram processo de clarificação com hipoclorito de sódio a $50 \%$. Todas as secções foram coradas com safranina a $1 \%$ e azul de anilina $1,0 \%$ e montadas em lâmina e lamínula com adesivo Entellan.

As lâminas foram observadas em microscópio óptico Leica, com uma câmera acoplada para realização das fotografias dos cortes. As fotos foram utilizadas para as medições dos parâmetros anatômicos pelo programa de análise de imagem QWin, calibrado com régua microscópica nos mesmos aumentos das fotografias, segundo metodologia descrita por Pereira et al. (2008).

Foram mensurados os seguintes parâmetros quantitativos dos tecidos: espessura da epiderme da face abaxial (EAB); espessura do mesofilo (MF); espessura do limbo (EL); espessura da epiderme da face adaxial (EAD); diâmetro do xilema (DX); e diâmetro do floema (DF), conforme demonstrado na Figura 1, de acordo com Carlquist (1975).

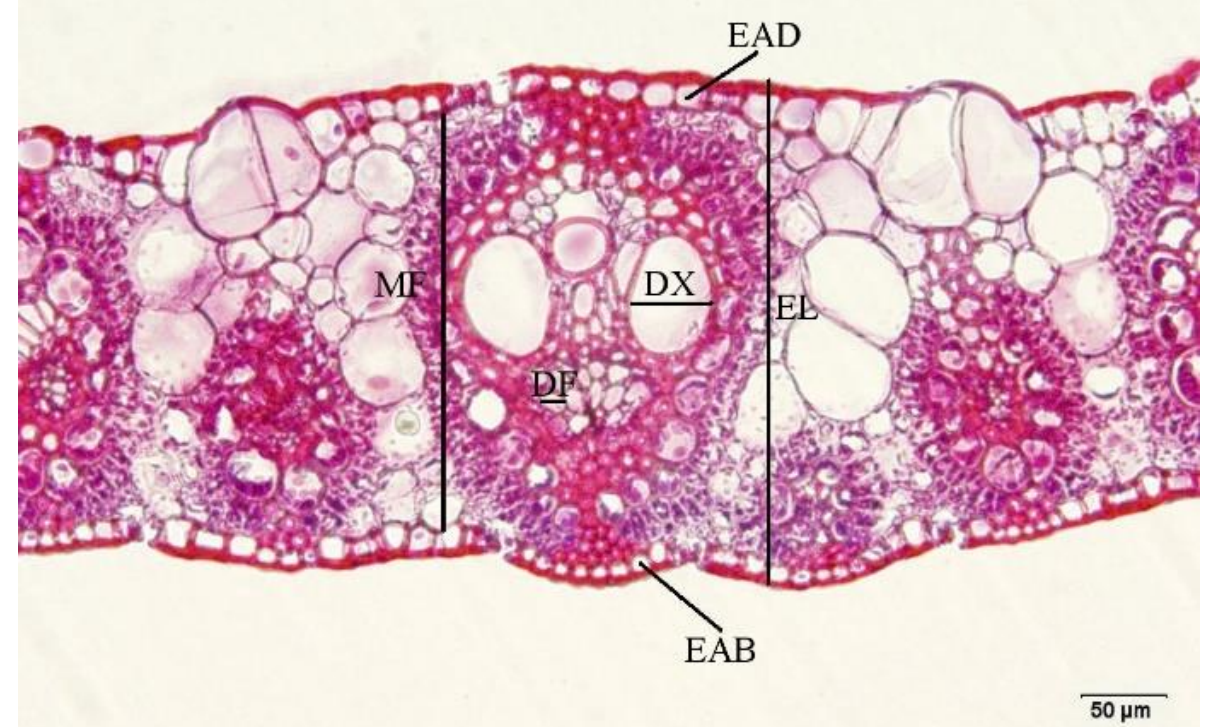

Figura 1. Características morfoanatômicas avaliadas nas folhas das variedades de cana-de-açúcar SP80-3280 e SP80-1842: epiderme da face abaxial (EAB); espessura do mesofilo (MF); espessura do limbo (EL); espessura da epiderme da face adaxial (EAD); espessura da;); diâmetro do xilema (DX); e diâmetro do floema (DF). Igaraçu do Tietê (SP), 2011.

As variáveis foram submetidas à análise de variância pelo teste $\mathrm{F}(\mathrm{p}<0,05)$ e ao teste de médias Tukey 5\% (Banzato e Kronka, 2006), sendo utilizado o programa Assistat 7.6 Beta (Silva e Azevedo, 2002).

\section{Resultados e Discussão}

Entre todas as características estudadas, apenas a espessura da epiderme da face abaxial, a espessura do mesofilo e a espessura do limbo apresentaram interação significativa 
entre os fatores estudados, ou seja, variedades e maturadores químicos, segundo demonstrado na Tabela 1.

Para a característica espessura da epiderme da face abaxial, na variedade SP803280 não houve diferença significativa entre os tratamentos. Para a variedade SP80-1842, o tratamento com aplicação de Ethrel (etephon) resultou em maiores valores para essa característica. Ainda para a característica espessura da epiderme da face abaxial, nos tratamentos com Ethrel (etephon) e Moddus (ethyl-trinexapac) a variedade SP80-1842 exibiu os maiores valores.

Tabela 1. Espessura da epiderme da face abaxial (EAB), espessura do mesofilo (MF) e espessura do limbo (EL) para a interação entre variedade de cana-de-açúcar plantada (SP80-3280 e SP801842) e maturadores químicos aplicados. Igaraçu do Tietê (SP), 2011.

\begin{tabular}{lcccccc}
\hline \multirow{2}{*}{ Trat. } & \multicolumn{2}{c}{ EAB $(\mathrm{mm})$} & \multicolumn{2}{c}{ MF $(\mathrm{mm})$} & \multicolumn{2}{c}{ EL $(\mathrm{mm})$} \\
\cline { 2 - 7 } & SP80-3280 & SP80-1842 & SP80-3280 & SP80-1842 & SP80-3280 & SP80-1842 \\
\hline C & $14,55 \mathrm{Aa}$ & $15,10 \mathrm{aAB}$ & $202,54 \mathrm{bA}$ & $233,7 \mathrm{aAB}$ & $5,52 \mathrm{bA}$ & $7,10 \mathrm{aAB}$ \\
$\mathrm{C}+\mathrm{R}$ & $13,58 \mathrm{aA}$ & $14,65 \mathrm{aAB}$ & $209,10 \mathrm{aA}$ & $230,1 \mathrm{aAB}$ & $6,53 \mathrm{aA}$ & $5,9 \mathrm{aB}$ \\
E & $12,69 \mathrm{bA}$ & $16,89 \mathrm{aA}$ & $225,36 \mathrm{bA}$ & $274,59 \mathrm{aA}$ & $5,57 \mathrm{bA}$ & $7,58 \mathrm{aA}$ \\
E+R & $13,83 \mathrm{aA}$ & $14,72 \mathrm{aAB}$ & $232,34 \mathrm{aA}$ & $239,8 \mathrm{aAB}$ & $6,39 \mathrm{aA}$ & $6,43 \mathrm{aAB}$ \\
M & $13,13 \mathrm{bA}$ & $16,25 \mathrm{aAB}$ & $233,21 \mathrm{aA}$ & $237,4 \mathrm{aAB}$ & $6,39 \mathrm{aA}$ & $7,06 \mathrm{aAB}$ \\
MTD & $14,59 \mathrm{aA}$ & $14,59 \mathrm{aAB}$ & $213,70 \mathrm{bA}$ & $269,9 \mathrm{aAB}$ & $6,81 \mathrm{aA}$ & $6,80 \mathrm{aAB}$ \\
MTD+R & $14,28 \mathrm{aA}$ & $15,35 \mathrm{aAB}$ & $219,66 \mathrm{aA}$ & $244,4 \mathrm{aAB}$ & $6,54 \mathrm{aA}$ & $6,08 \mathrm{aAB}$ \\
R & $14,24 \mathrm{aA}$ & $15,02 \mathrm{aAB}$ & $230,06 \mathrm{aA}$ & $240,5 \mathrm{aAB}$ & $5,84 \mathrm{bA}$ & $6,86 \mathrm{aAB}$ \\
Test & $14,14 \mathrm{aA}$ & $14,20 \mathrm{aB}$ & $241,24 \mathrm{aA}$ & $224,8 \mathrm{aB}$ & $5,94 \mathrm{aA}$ & $6,63 \mathrm{aAB}$ \\
\hline CV\% Varied. & \multicolumn{3}{c}{17,70} & \multicolumn{3}{c}{22,34} \\
DMS Varied. & \multicolumn{3}{c}{30,22} & \multicolumn{3}{c}{20,97} \\
CV\% Prod. & 1,53 & \multicolumn{2}{c}{18,06} & \multicolumn{3}{c}{1,53} \\
DMS Prod. & \multicolumn{3}{c}{48,20} & \multicolumn{3}{c}{6,44} \\
Média & 2,42 & \multicolumn{2}{c}{233,49} & \multicolumn{3}{c}{} \\
\hline
\end{tabular}

Médias seguidas pela mesma letra não diferem estatisticamente entre si pelo Teste de Tukey ao nível de 5\% de probabilidade. Letras maiúsculas representam diferenças nas colunas e letras minúsculas nas linhas. C: sulfometuron-methyl, C+G: sulfometuron-methyl + glyphosate, E: etephon, E+G: etephon + glyphosate, ET: ethyl-trinexapac, CC: Compostos de radicais carboxílicos orgânicos, CC+G: Compostos de radicais carboxílicos orgânicos - CC + glyphosate, G: glyphosate, Test: Testemunha.

Para a característica espessura do mesofilo, na variedade SP80-3280 também não houve diferença significativa entre os tratamentos. Para a variedade SP80-1842, o tratamento com aplicação de Ethrel (etephon) resultou em maiores valores. Ainda para a característica espessura do mesofilo, nos tratamentos com Curavial (sulfometuronmethyl), Ethrel (etephon) e MTD (Compostos de radicais carboxílicos orgânicos) a variedade SP80-1842 exibiu novamente os maiores valores.

Para a característica espessura do limbo, na variedade SP80-3280, mais uma vez, não houve diferença significativa entre os tratamentos. Para a variedade SP80-1842, o tratamento com aplicação de Ethrel (etephon) resultou em maiores valores para essa característica. Nos tratamentos com Curavial (sulfometuron-methyl), Ethrel (etephon) e Roundup (glyphosate) a variedade SP80-1842 exibiu os maiores valores para essa característica, conforme apresentado na Tabela 1 .

O uso de maturadores em cana-deaçúcar pode provocar diversas reações morfofisiológicas, afetando, dessa forma, o metabolismo vegetal. Castro et al. (2009) afirmam que estresses de qualquer natureza, sejam de origem biótica ou abiótica, podem ocasionar alterações na espessura epidérmica foliar. Plantas que apresentam epidermes mais espessas podem melhor proteger o mesofilo contra as diversas injúrias, já que as funções principais desse tecido são a proteção da superfície vegetal e o controle das trocas gasosas, conforme Korndörfer et al. (2002). Alterações morfológicas decorrentes de fatores 
estressantes foram relatados por Castro et al. (2007), que encontraram em plantas de Marcetia taxifolia, uma diminuição do tamanho das células epidérmicas abaxiais devido à deficiência hídrica.

Os resultados são discordantes daqueles encontrados por Figueiredo et al. (2013), que observaram que o aumento do estresse na planta acarretou em uma diminuição da espessura do mesofilo e do limbo da cana-deaçúcar. Queiroz-Voltan et al. (2011) constataram que as variações no mesofilo podem interferir potencialmente na capacidade fotossintética.

Para as características espessura da epiderme da face adaxial, diâmetro do xilema e diâmetro do floema, não houve interação significativa entre variedades e maturadores químicos, segundo demonstrado na Tabela 2. No entanto, houve diferença significativa para os fatores isolados.

Tabela 2. Espessura da epiderme da face adaxial (EAD), diâmetro do xilema (DX) e diâmetro do floema (DF) para as variedades de cana-de-açúcar plantadas e para cada um dos maturadores químicos aplicados. Igaraçu do Tietê, SP, 2011.

\begin{tabular}{lccc}
\hline & EAD $(\mathrm{mm})$ & $\mathrm{DX}(\mathrm{mm})$ & $\mathrm{DF}(\mathrm{mm})$ \\
\hline SP80-3280 & $14,73 \mathrm{~b}$ & $44,59 \mathrm{a}$ & $7,47 \mathrm{a}$ \\
SP80-1842 & $15,34 \mathrm{a}$ & $42,00 \mathrm{~b}$ & $7,70 \mathrm{a}$ \\
\hline DMS & 0,47 & 2,31 & 0,46 \\
\hline $\mathrm{C}$ & $15,58 \mathrm{abc}$ & $39,08 \mathrm{~b}$ & $7,17 \mathrm{a}$ \\
$\mathrm{C}+\mathrm{R}$ & $14,28 \mathrm{bc}$ & $44,41 \mathrm{ab}$ & $7,91 \mathrm{a}$ \\
$\mathrm{E}$ & $16,38 \mathrm{a}$ & $43,61 \mathrm{ab}$ & $7,84 \mathrm{a}$ \\
$\mathrm{E}+\mathrm{R}$ & $15,15 \mathrm{abc}$ & $43,82 \mathrm{ab}$ & $7,21 \mathrm{a}$ \\
M & $15,78 \mathrm{ab}$ & $44,76 \mathrm{ab}$ & $7,41 \mathrm{a}$ \\
MTD & $14,54 \mathrm{bc}$ & $41,94 \mathrm{ab}$ & $7,99 \mathrm{a}$ \\
MTD+R & $14,80 \mathrm{abc}$ & $46,79 \mathrm{a}$ & $7,91 \mathrm{a}$ \\
R & $14,78 \mathrm{abc}$ & $43,85 \mathrm{ab}$ & $7,03 \mathrm{a}$ \\
Test & $14,01 \mathrm{c}$ & $7,78 \mathrm{a}$ \\
\hline DMS & 1,74 & $41,37 \mathrm{ab}$ & 1,50 \\
\hline Médias seguidas pela mesma letra não diferem estatisticamente entre si pelo Teste de Tukey ao nível de 5\% de probabilidade. C: sulfometuron- & - \\
methyl, C+G: sulfometuron-methyl + glyphosate, E: etephon, E+G: etephon + glyphosate, ET: ethyl-trinexapac, CC: Compostos de radicais \\
carboxílicos orgânicos, CC+G: Compostos de radicais carboxílicos orgânicos - CC + glyphosate, G: glyphosate, Test: Testemunha.
\end{tabular}

Para a característica espessura da epiderme da face adaxial, a variedade SP801842 apresentou os maiores valores. De maneira geral, o tratamento com Ethrel (etephon) proporcionou maiores valores para essa característica, nas duas variedades estudadas.

Para a característica diâmetro do xilema, a variedade SP80-3280 apresentou os maiores valores. Para essa característica, nas duas variedades, o tratamento com MTD + Roundup (Compostos de radicais carboxílicos orgânicos + glyphosate) proporcionou maiores valores. Entretanto, o tratamento com Curavial (sulfometuron-methyl) provocou uma diminuição no diâmetro do xilema.
Para a característica diâmetro do floema, os resultados não apresentaram diferenças significativas em nenhum dos fatores estudados.

Plantas com epidermes foliares mais espessas permitem uma melhor adaptação às condições adversas e melhores chances de sobrevivência. Segundo Castro et al. (2009) e Faria et al. (2014), a diminuição da espessura da epiderme foliar conduz a planta à uma condição de fragilidade em relação aos fatores do meio, tanto em relação ao ataque de pragas e patógenos quanto à sua capacidade de reflexão da radiação luminosa. $O$ fato é decorrente da presença da cutícula, que além de outras funções, previne o superaquecimento 
provocado pela radiação infravermelha proveniente dos raios solares.

Conforme citado anteriormente, a aplicação de maturadores químicos em canade-açúcar pode promover alterações morfofisiológicas nas plantas, em decorrência das modificações provocas no ambiente produtivo. Os resultados corroboram com Cunha et al. (2008), que estabelecem uma relação direta entre a modificação das características do meio e as alterações anatômicas das células dos tecidos vegetais. Os autores verificaram, ainda, uma maior lignificação e diminuição do diâmetro do tecido vascular, quando da aplicação de cádmio e zinco.

Figueiredo et al. (2013) explica que os menores diâmetros dos vasos condutores podem influenciar de forma direta $\mathrm{e}$ significativa na fotossíntese, consequentemente no crescimento e desenvolvimento dos órgãos vegetais.

A anatomia vegetal pode indicar características que atribuem tolerância a diferentes condições ambientais para as plantas cultivadas, como seca, alagamento ou qualidade e intensidade da radiação que incide sobre as folhas, podendo promover modificações na espessura do mesofilo, nos tecidos vasculares, na espessura da epiderme, na espessura da cutícula e densidade estomática, segundo Ribeiro et al. (2012) e Pincelli e Silva (2012).

Os resultados encontrados no presente trabalho são concordantes com Ferreira (2005) e Ramos et al. (2014), que relataram que variedades de cana-de-açúcar, quando cultivadas nas mesmas condições, podem apresentar diferentes proporções de tecidos vegetais, em decorrência da capacidade genética de cada cultivar.

O efeito residual da aplicação de maturadores na cana-de-açúcar pode provocar diferentes alterações morfoanatômicas foliares, com efeitos positivos ou negativos, que podem influenciar no crescimento e desenvolvimento das plantas.
Em função disso, as modificações morfológicas de cada cultivar, assim como os efeitos provocados por elas, devem ser cada vez mais estudadas, a fim de melhorar o entendimento e direcionamento das pesquisas pertinentes.

\section{Conclusões}

De maneira geral, as características morfoanatômicas foliares de cana-de-açúcar apresentaram maiores valores médios para o efeito residual do maturador Ethrel (etephon). A variedade SP80-1842 exibiu os maiores valores para a maioria das características estudadas.

\section{Referências}

Banzato, D.A.; Kronka, S.N. Experimentação agrícola. 3.ed. Jaboticabal: FUNEP, 2006. $237 p$.

Benedini, M.S.; Ricci Júnior, A. Respostas das variedades CTC a maturadores. Revista Coplana, fev. 2009.

Carlquist,S. Ecological estrategies of xylem evolution.Berkeley: University of California Press, 1975. 259 p.

Caputo, M.M.; Beauclair, E.G.F.; Silva, M.A.; Piedade, S.M.S. Resposta de genótipos de cana-de-açúcar à aplicação de indutores de maturação. Bragantia, v.67, n.1, p.15-23, 2008.

Castro, E.M.; Pereira, F.J.; Paiva, R. Histologia vegetal: estrutura e função de órgãos vegetativos. Lavras: Universidade Federal de Lavras, 2009. 234p.

Castro, E.M.; Pinto, J.E.B.P.; Soares, A.M.; Melo, H.C.; Bertalucci, S.K.V.; Vieira, C.V. et al. Adaptações anatômicas de folhas de Mikania glomerata Sprengel (Asteraceae), em três regiões distintas da planta, em diferentes níveis de sombreamento. Revista Brasileira de Plantas Medicinais, v.9, n.2, p.8-16, 2007. 
Cunha, K.P.V.; Nascimento, C.W.A.; Pimentel, R.M.M.; Accioly, A.M.A.; Silva, A.J.S. Disponibilidade, acúmulo e toxidez de cádmio e zinco em milho cultivado em solo contaminado. Revista Brasileira de Ciência do Solo, v.32, n.3, p.1319-1328, 2008.

Embrapa. Centro Nacional de Pesquisa dos Solos. Sistema brasileiro de classificação de solos. Rio de Janeiro: EMBRAPA/CNPS, 2013. 353p.

Faria, A.T.; Silva, A.F.; Ferreira, E.A.; Rocha, P.R.R.; Silva, D.V.; Silva, A.A. et al. Alterações nas características fisiológicas da cana-de-açúcar causadas por trinexapac-ethyl. Revista Brasileira Ciências Agrárias, v.9, n.2, p.200-204, 2014.

Ferreira, E.A. Anatomia quantitativa, micromorfologia e sensibilidade a herbicidas em genótipos de cana-de-açúcar. 2005. 73f. Dissertação (Mestrado em Botânia) Universidade Federal de Viçosa, Viçosa-MG, 2005.

Figueiredo, P.A.M.; Ramos, S.B.; Viana, R.S.; Lisboa, L.A.M.; Heinrichs, R. Alterações morfoanatômicas foliares da cana-de-açúcar na fase de estabelecimento em condições de matocompetição. Planta Daninha, v.31, n.4, p.777-784, 2013.

Korndörfer, G.H.; Pereira, H.S.; Camargo, M.S. Papel do silício na produção de cana-deaçúcar. STAB, v.21, n.2, p.6-9, 2002.

Kraus, J.E.; Arduim, M. Manual básico em métodos em morfologia vegetal. Rio de Janeiro: EDU, 1999. p.221.

Leite, G.H.; Crusciol, C.A.C.; Silva, M.A. Desenvolvimento e produtividade da cana-deaçúcar após aplicação de reguladores vegetais em meio de safra. Semina: Ciências Agrárias, v.32, n.1, p.129-138, 2011.

Leite, G.H.P.; Crusciol, C.A.C.; Silva, M.A.; Lima, G.P.P. Atividade das enzimas invertases e acúmulo de sacarose em cana-de-açúcar sob efeito do nitrato de potássio, etefon e etil- trinexapac. Ciência e Agrotecnologia, v.35, n.4, p.649-656, 2011.

Leite, G.H.P.; Crusciol, C.A.C.; Silva, M.A.S.; Filho, W.G.V. Qualidade tecnológica da canade-açúcar em função da aplicação de maturadores em meio de safra. Bragantia, v.68, n.2, p.527-534, 2009.

Pereira, F.J.; Castro, E.M.; Souza, T.C.; Magalhães, P.C. Evolução da anatomia radicular do milho 'Saracura' em ciclos de seleção sucessivos. Pesquisa Agropecuária Brasileira, v.43, n.12, p.1649-1656, 2008.

Pincelli, R.P.; Silva, M.A. Alterações morfológicas foliares em cultivares de cana-deaçúcar em resposta à deficiência hídrica. Bioscience Journal, v.28, n.4, p.546-556, 2012.

Queiroz-Voltan, R.B.; Rolim, G.S.; Pedro Júnior, M.J.; Hernandes, J.L. Variações na anatomia foliar de videira Niagara em diferentes sistemas de condução. Bragantia, v.70, n.3, p.488-493, 2011.

Ramos, S.B.; Viana, R.S.; Ventura, G.; Segati, D.F.; Assumpção, A.C.N.D.; Fruchi, V.M. et al. Características morfoanatômicas foliares de cultivares de cana-de-açúcar. STAB. v.32 n.4. p.28-30, 2014.

Ribeiro, M.N.O.; Carvalho, S.P.; Pereira, F.J.; Castro, E. M. Leaf anatomy of the cassava as related to potential for tolerance to different environmental conditions. Revista Ciência Agronômica, v.43, n.2, p.354-361, 2012.

Scarpari, M.S.; Beauclair, E.G.F. Sugarcane maturity estimation through edaphic-climatic parameters. Scientia Agrícola, v.61, n.5, p.486-491, 2004.

Silva, D.P. Thiametoxam em cana-de-açúcar manejada com maturadores. 2012. 61f. Dissertação (Trabalho de Mestrado em Agronomia) - Faculdade de Ciências Agronômicas da UNESP - Campus de Botucatu, 2012. 
Silva, F.A.S.; Azevedo, C.A.V. Versão do programa computacional Assistat para o sistema operacional Windows. Revista Brasileira de Produtos Agroindustriais, v.4, n.1, p.71-78, 2002.

Silva, P.V.; Monquero, P.A.; Munhoz, W.S. Controle em pós-emergência de plantas daninhas por herbicidas utilizados na cultura da cana-de-açúcar. Revista Caatinga, v.28, n.4, p.21-32, 2015. 